\title{
Particle finite element method in fluid-mechanics including thermal convection-diffusion
}

\author{
R. Aubry ${ }^{a}$, S.R. Idelsohn ${ }^{\text {b,* }}$, E. Oñate ${ }^{\text {a }}$ \\ ${ }^{a}$ International Center for Numerical Methods in Engineering (CIMNE), Universidad Politécnica de Cataluña, Barcelona, Spain \\ ${ }^{\mathrm{b}}$ International Center for Computational Methods in Engineering (CIMEC) Universidad Nacional del Litoral and CONICET, \\ Guemes 3450, 3000 Santa Fe, Argentina
}

Accepted 27 October 2004

\begin{abstract}
A method is presented for the solution of an incompressible viscous fluid flow with heat transfer using a fully Lagrangian description of the motion. Due to the severe element distortion, a frequent remeshing is performed in an efficient manner. An implicit time integration through a classical fractional step is presented. The non-linearities of the formulation are taken into account and solved with the fixed-point iteration method. The displacement and temperature solutions are coupled through the Boussinesq approximation. The Lagrangian formulation provides an elegant way of solving free-surface problems with thermal convection as the particles are followed during their motion. To illustrate the method, the Rayleigh-Bénard instability with and without free surface in two dimensions has been computed.
\end{abstract}

(c) 2005 Elsevier Ltd. All rights reserved.

Keywords: Particle method; Lagrangian description; Coupled thermo-mechanical analysis; Thermal convection; Rayleigh-Bénard instability with free surface; Incompressible fluid flow

\section{Introduction}

The classical Navier-Stokes equations have been studied for a long time, both from the theoretical and numerical points of view. These equations were mostly associated with an Eulerian description of motion which led to the impressive results in computational fluid

\footnotetext{
${ }^{*}$ Corresponding author. Address: International Center for Numerical Methods in Engineering (CIMNE), Universidad Politécnica de Cataluña, Barcelona, Spain. Tel.: +54 342 4511595; fax: +54 3424511169 .

E-mail addresses: sergio@ceride.gov.ar (S.R. Idelsohn), onate@cimne.upc.es (E. Oñate).
}

dynamics (CFD) during the last 20 years. Surprisingly, the Lagrangian description was much less exploited, despite of the advantage of this method for certain types of problems like free surface tracking [37] and fluid-structure interaction including heat transfer effects. The first problem dealing with a Lagrangian formulation for fluids is the need for a constant remeshing due to the severe distortion of the mesh as the nodes move in time. Particle type methods offer an appealing alternative to this remeshing and have been used extensively. A precursor in this field was Monoghan [19] for the treatment of astrophysical hydrodynamic problem with the so called Smooth Particle Hydrodynamics Method (SPH). Kernel approximations are used in the SPH method to interpolate the unknowns. 
On the other hand, meshless methods have been developed both for structural $[2,13]$ and fluid mechanical problems $[33,34]$. All these methods use the idea of a polynomial interpolant that fits a number of points minimizing the distance between the interpolated function and the values of the unknown point. Meshless methods however still require to compute the nodal connectivities of each 'star' node in a cloud of nodes and this can be a costly task. Lately the meshless ideas were generalized to derive finite element type approximations where meshes are generated in a computing time of the order of the number of nodes in the mesh [23].

In this paper, a Lagrangian method will be used together with a particular form of the FEM, the Particle finite element method (PFEM) [25,32] in order to solve thermal convection for incompressible fluid flows. The method combines the best features of particle (meshless) techniques and finite element methods. In the first part, the basis of the Lagrangian and Eulerian descriptions are briefly presented. In the second part, the heat and Navier-Stokes equations and their weak expressions are derived in a form suitable for the non-linear coupled problem. The discretization of these equations in space and time is then discussed. A fractional scheme for the timge integration of the equations of motion using the finite element method is presenter ization of the equations

coupled terms induced by the Bou given. After a few computational stability, reference configu
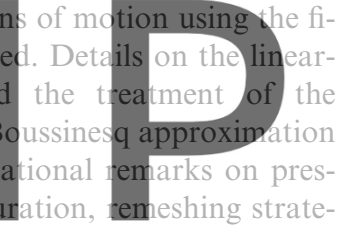

gies, and particle behaviour of the method, numerical

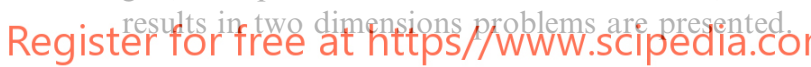

\section{Lagrangian versus Eülerian formulation}

In this section, the main features of the Lagrangian and Eulerian approaches are recalled, the notations are defined and the incompressibility condition is derived in both formulations.

\subsection{A few relations between Lagrangian and Eulerian tensor derivatives}

In the Lagrangian formulation, the notions of reference and current configuration play a central part [35]. The Lagrangian formulation describes all the measured quantities with respect to the position of a particle on a previously chosen reference configuration and to time, as $M=M(\mathbf{X}, t)$ where $M$ is whatever interesting quantity and $\mathbf{X}$ the reference position. On the other hand, the Eulerian description uses the position $\mathbf{x}$ of a given particle on the current configuration to define the quantity $M=m(\mathbf{x}, t)$. Quantities in the reference and current configuration will be written with capital and lower case letters, respectively. Obviously, both should describe the same property, which is noted introducing the configuration as a mapping of the location of a particle $\mathbf{X}$ of a body into $\mathscr{R}^{n}$ as $\mathbf{x}=\mathscr{X}(\mathbf{X}, t)$, writing

$M=m(\mathbf{x}, t)=m(\mathscr{X}(\mathbf{X}, t), t)=M(\mathbf{X}, t)$

All tensor fields and their derivatives will be written with respect to a particular reference. A classical issue is to relate a derivative of a scalar tensor $M$ in the Eulerian and Lagrangian descriptions. There, the deformation gradient $\mathbf{F}$, plays an important role

$\mathbf{F}=\operatorname{grad}_{X}\left(\mathscr{X}(\mathbf{X}, t)=\operatorname{grad}_{X}(\mathbf{x})\right.$

Using differential calculus, one gets

$\frac{\partial m(x, t)}{\partial x}=\frac{\partial M(X, t)}{\partial X} \frac{\partial X}{\partial x}$

which leads, for a scalar quantity $M$, to

$\operatorname{grad}_{x}(m)=\mathbb{F}^{-\mathrm{T}} \operatorname{grad}_{X}(M)$

and, for a first-order tensor $\mathbf{V}$

$\operatorname{grad}_{x}(\mathbf{v})=\operatorname{grad}_{X}(\mathbf{V}) \mathbf{F}^{-1}$

The well-known Nanson's formula is helpful to get a similar relation for the divergence of a second-order

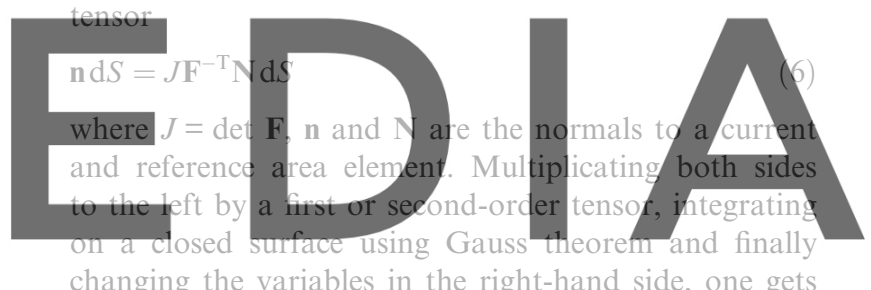

changing the variables in the right-hand side, one gets o dovindorobthe version without the watermark

$\operatorname{div}_{x}(\mathbf{v})=\frac{1}{J} \operatorname{div}_{X}\left(J \cdot \mathbb{F}^{-1} \mathbf{V}\right)$

and for a second-order tensor $\boldsymbol{\sigma}$

$\operatorname{div}_{x}(\boldsymbol{\sigma})=\frac{1}{J} \operatorname{div}_{X}\left(J \boldsymbol{\sigma} \cdot \mathbf{F}^{-\mathrm{T}}\right)$

These relations are classically known as the Piola transform of a tensor [28]. Finally, differentiating $\mathbf{V}=\mathbf{V}(\mathbf{X}, t)=\mathbf{v}(\mathscr{X}(\mathbf{X}), t)$ with respect to time leads to

$\frac{\mathrm{DV}}{\mathrm{D} t}=\frac{\partial \mathbf{v}}{\partial t}+\mathbf{v} \cdot \operatorname{grad}_{x}(\mathbf{v})$

The first term refers to the material derivative of a vector $\mathbf{V}$ and the second one to the spatial derivative. In a Lagrangian formulation the convective term is implicitely contained in the material derivative as the velocities are function of the material particles.

\subsection{The incompressibility condition in the Lagrangian and Eulerian formulations}

The conservation of mass and the incompressibility condition are good examples of how to switch from 
the Lagrangian to the Eulerian formulation and viceversa. Writing the conservation of mass between two instants in two different configurations and changing the variables between both configurations, one obtains

$\rho_{0}=\rho J$

where $\rho$ and $\rho_{0}$ are the mass densities in the current and reference configuration. The last equation describes the conservation of mass in the Lagrangian formulation. Differentiating (10) with respect to time leads to

$\dot{\rho} J=-\rho \dot{J}$

Using the fact that

$\dot{J}=J \operatorname{div}_{x}(\mathbf{v})$

one gets

$-\frac{\dot{\rho}}{\rho}=\frac{\dot{J}}{J}=\operatorname{div}_{x}(\mathbf{v})$

which is the classical mass conservation in the Eulerian formulation. The incompressibility condition in a Lagrangian frame is thus derived

$\rho=\rho_{0} \quad$ or $\quad J=1$

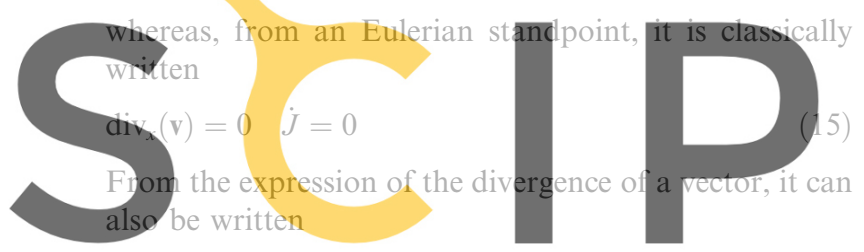

$\operatorname{Tr}\left(\operatorname{grad}_{X}(\mathbf{V}) \mathbb{F}^{-1}\right)=0$

(16)

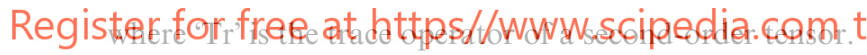

This expression will be used later on.

\section{The heat and Navier-Stokes equations in the Lagrangian formulation}

The results of the preceding section are now used to derive the heat equation and the Navier-Stokes equations in the Lagrangian formulation. These equations will be the foundations of the numerical method presented in the next section.

\subsection{The heat equation}

In this paragraph, the strong form of the heat equation in a Lagrangian formulation will be obtained from the well known Eulerian formulation. Then, the variational form will be derived and the relationships between both formulations will be emphasized.

\subsubsection{Strong form of the heat equation}

There are several ways to introduce the Lagrangian formulation, either directly from the conservation prin- ciples or from the classical Eulerian equations. The second way will be chosen as these equations are very well-known. Writing the classical heat equation with convection and a source term in an Eulerian form, one obtains

$\rho C \partial_{t} T+\rho C\left(\mathbf{v} \cdot \operatorname{grad}_{x}(T)\right)=\operatorname{div}_{x}\left(\kappa \operatorname{grad}_{x}(T)\right)$

where $T$ is the temperature, $\kappa$ the thermal conductivity, $\rho$ the density, $C$ the heat capacity and $\mathbf{v}$ represents the convective velocity. As seen before, the term in the right hand side represents the spatial derivative. Using the material derivative and the Piola transform, the heat equation in the Lagrangian description reads

$\rho_{0} C \frac{\mathrm{D} T}{\mathrm{D} t}=\operatorname{div}_{X}\left(\kappa J \mathbf{F}^{-1} \mathbf{F}^{-\mathrm{T}} \operatorname{grad}_{X}(T)\right)$

Here, the same notation has been used for the representation of the temperature in an Eulerian and in a Lagrangian descriptions for the sake of simplicity.

Remark 3.1. No convective term appears in the last equation which has a linear appearance. However, the non-linearity of this equation comes from the fact that F, the deformation gradient, depends on the displacement U. As noted in [41], the Lagrangian equations are

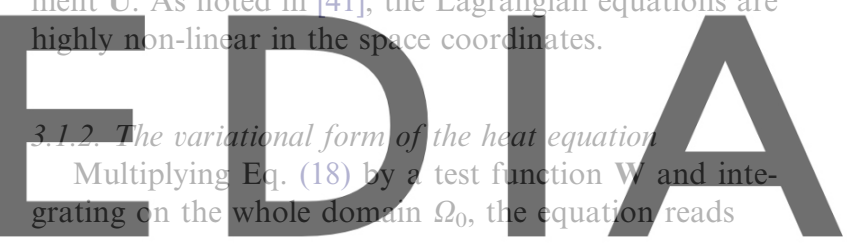

$\int \rho_{0} C \frac{\mathrm{D} T}{\mathrm{D} t} \mathbf{W} \mathrm{d} V_{0}=\int \operatorname{div}_{X}\left(k J \mathbb{F}^{-1} \mathbb{F}^{-\mathrm{T}} \operatorname{grad}_{X}(T)\right) \mathbf{W} \mathrm{d} V_{0}$ download the version without the watermark

Integrating by parts the right term of Eq. (19) leads to

$$
\begin{aligned}
& \int_{\Omega_{0}} \rho_{0} C \frac{\mathrm{D} T}{\mathrm{D} t} \mathbf{W} \mathrm{d} V_{0} \\
& =-\int_{\Omega_{0}} \kappa J \mathbf{F}^{-1} \mathbf{F}^{-\mathrm{T}} \operatorname{grad}_{X}(T) \cdot \operatorname{grad}_{X}(\mathbf{W}) \mathrm{d} V_{0}
\end{aligned}
$$

\subsection{The Navier-Stokes equations}

The same structure as the preceeding part is followed to derive the Lagrangian form of the Navier-Stokes equations. First, the strong formulation is presented through the introduction of the first Piola-Kirchhoff stress tensor. Then, the variational formulation is derived and the link between Eulerian and Lagrangian formulation is also highlighted.

\subsubsection{Strong form of the Navier-Stokes equations}

In the Eulerian frame, the classical equation of momentum conservation reads

$\rho \partial_{t} \mathbf{v}+\rho \mathbf{v} \cdot \operatorname{grad}_{x} \mathbf{v}=\operatorname{div}_{x}(\boldsymbol{\sigma})+\rho \mathbf{f}$ 
where $\mathbf{v}$ is the velocity, $\mathbf{f}$ an external force per unit of mass, and the stresses $\boldsymbol{\sigma}$ are related to the pressure and the velocities by

$\boldsymbol{\sigma}=-p \mathbf{I}+2 \mu \mathbf{D}$

for a Newtonian fluid where, in Eq. (22), $\mu$ is the fluid viscosity and $\mathbf{D}$ is the symmetric part of the gradient velocity, referred to the deformed configuration. Furthermore, considering the incompressibility condition, the classical Navier-Stokes equations read

$\rho\left(\partial_{t} \mathbf{v}+\mathbf{v} \cdot \operatorname{grad}_{x}(\mathbf{v})\right)=-\operatorname{grad}_{x}(p)+\mu \Delta \mathbf{v}+\rho \mathbf{f}$

$\operatorname{div}_{x}(\mathbf{v})=0$

In the reference configuration, using the Piola transform for the second-order stress tensor $\sigma$ leads to

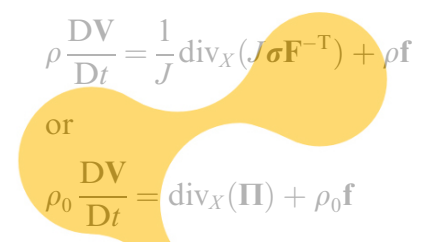

where IT is the first Piola-Kirchhoff stress tensor defined

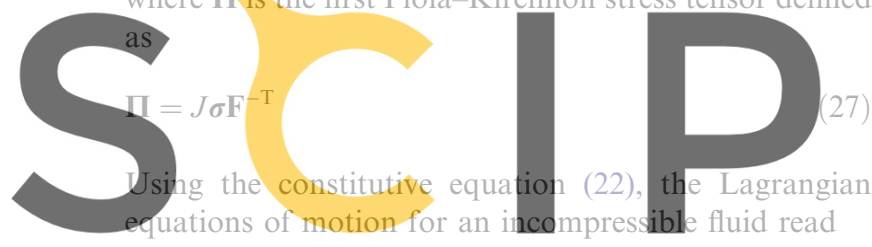

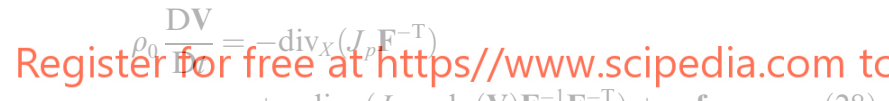
$+\mu \operatorname{div}_{X}\left(J \operatorname{grad}_{X}(\mathbf{V}) \mathbf{F}^{-1} \mathbf{F}^{-\mathrm{T}}\right)+\rho_{0} \mathbf{f}$

(28)

$\operatorname{Tr}\left(\operatorname{grad}_{X}(\mathbf{V}) \mathbf{F}^{-1}\right)=0$

Remark 3.2. As in the heat equation, the non-linearity of these equations appears through the deformation gradient. Both the velocity and the displacement appear in these equations, coupled with the pressure.

\subsubsection{The mixed variational form of the Navier-Stokes equations}

Eqs. (28) and (29) are multiplied by test functions $\mathbf{W}$ and $q$, respectively. The variational form of the Lagrangian Navier-Stokes equations reads, after integration by parts of these equations

$$
\begin{aligned}
\int_{\Omega_{0}} & \rho_{0} \\
= & \int_{\Omega_{0}} J_{p} \mathbf{F}^{-\mathrm{T}} \cdot \operatorname{grad}_{X}(\mathbf{W}) \mathrm{d} V_{0} \\
& -\int_{\Omega_{0}} \mu J \operatorname{grad}_{X}(\mathbf{V}) \mathbf{F}^{-1} \mathbf{F}^{-\mathrm{T}}: \operatorname{grad}_{X}(\mathbf{W}) \mathrm{d} V_{0}
\end{aligned}
$$

$$
\int_{\Omega_{0}} J \operatorname{Tr}\left(\operatorname{grad}_{X}(\mathbf{V}) \mathbf{F}^{-1}\right) q \mathrm{~d} V_{0}=0
$$

\section{Discretization of the equations}

In this section, the equations derived in the last section are discretized. A classical time discretization is first proposed. The space discretization is then described, and a fractional step introduced. After the linearization of the equations, the thermo-mechanical scheme is finally derived.

\subsection{Discretization in time}

Consider a Newmark scheme [22]

$$
\mathbf{V}^{n+1}=\mathbf{V}^{n}+\delta t\left(\theta \mathbf{A}^{n+1}+(1-\theta) \mathbf{A}^{n}\right)
$$

$$
\mathbf{U}^{n+1}=\mathbf{U}^{n}+\delta t \mathbf{V}^{n}+\frac{\delta t^{2}}{2}\left(2 \beta \mathbf{A}^{n+1}+(1-2 \beta) \mathbf{A}^{n}\right)
$$

where $\theta$ and $\beta$ are two numerical parameters which determine the stability and accuracy of the algorithm. Applying the Newmark scheme to the weak form of

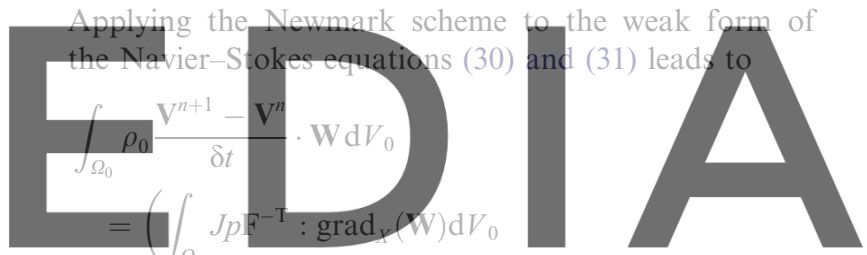

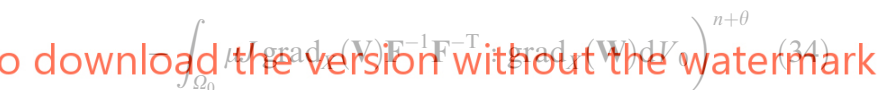
with

$\int_{\Omega_{0}} J \operatorname{Tr}\left(\operatorname{grad}_{X}\left(\mathbf{V}^{n+1}\right) \mathbf{F}^{-1}\right) q \mathrm{~d} V_{0}=0$

where

$X^{n+\theta}=\theta X^{n+1}+(1-\theta) X^{n}$

The values of $\theta$ and $\beta$ determine the order of the method. However, as seen in the following sections, the order of the method is limited to 1 so that values of $\theta=1$ and $\beta=0.25$ are chosen.

\subsection{Finite element discretization}

The velocity, the displacement and the pressure are discretized in the standard finite element manner as

$V_{j}=\sum N_{i}(X, t) V_{i j} \quad U_{j}=\sum N_{i}(X, t) U_{i j}$

$p=\sum N_{i}(X, t) P_{i}$

where the $N_{i}$ are the nodal shape functions. Substituting the finite element approximation (37) into the 
variational equation (30) and choosing a Galerkin formulation $\left(W_{i}=N_{i}\right)$ leads to the following system of equations:

$$
\frac{\mathbf{M}}{\delta t}\left(\mathbf{V}^{n+1}-\mathbf{V}^{n}\right)+\mathbf{K}\left(\mathbf{U}^{n+\theta}\right) \mathbf{V}^{n+\theta}+\mathbf{G}\left(\mathbf{U}^{n+\theta}\right) \mathbf{P}^{n+\theta}=\mathbf{F}^{n+\theta}
$$

$$
\mathbf{D}\left(\mathbf{U}^{n+\theta}\right) \mathbf{V}^{n+1}=0
$$

where the previous matrices are

$$
\begin{aligned}
M_{i j}^{a b} & =\delta_{a b} \int_{\Omega_{0}} \rho_{0} N_{i} N_{j} \mathrm{~d} V_{0} \\
K_{i j}^{a b} & =\delta_{a b} \int_{\Omega_{0}} \mu J \operatorname{grad}_{X}\left(N_{i}\right) \mathbf{F}^{-1} \cdot \operatorname{grad}_{X}\left(N_{j}\right) \mathbf{F}^{-1} \mathrm{~d} V_{0}
\end{aligned}
$$

$$
\mathrm{G}_{i j}=-\int_{\Omega_{0}} J \mathrm{~F}^{-\mathrm{T}} \operatorname{grad}_{X}\left(N_{i}\right) N_{j} \mathrm{~d} V_{0}
$$

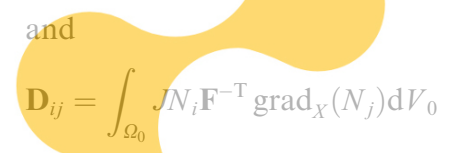

where the subscripts refer to the node indexes and the superscripts to the space indexes. Remark 4.1. The mass matrix linear dependance with $\mathbf{U}^{n+1}$ assembled on the last known nies e also function of $\mathbf{U}^{n+1}$. This
ritten for the sake of clarity.

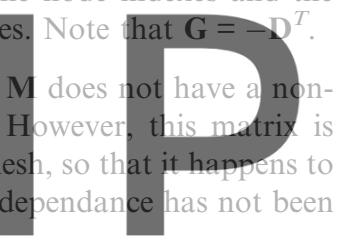

Register for free at https/fwwww.scipedia.com to

At this point, the classical fractional step method is introduced for the solution in time of Eqs. (38) and (39) as proposed in [9] and originated in [40] at the continuous level. However, the approach followed here is the one chosen in [9] so that the fractional step is presented at the algebraic level as a matrix manipulation. An auxiliary variable $\widetilde{\mathbf{V}}$ is introduced, representing the intermediate velocity. An equivalent problem to Eqs. (38) and (39) reads

$$
\begin{aligned}
& \frac{\mathbf{M}}{\delta t}\left(\widetilde{\mathbf{V}}^{n+1}-\mathbf{V}^{n}\right)+\mathbf{K}\left(\mathbf{U}^{n+\theta}\right) \widetilde{\mathbf{V}}^{n+\theta}+\gamma \mathbf{G}\left(\mathbf{U}^{n+\theta}\right) \mathbf{P}^{n}=\mathbf{F}^{n+\theta} \\
& \frac{\mathbf{M}}{\delta t}\left(\mathbf{V}^{n+1}-\widetilde{\mathbf{V}}^{n+1}\right)+\mathbf{G}\left(\mathbf{U}^{n+\theta}\right)\left(\mathbf{P}^{n+\theta}-\gamma \mathbf{P}^{n}\right)=0 \\
& \mathbf{D}\left(\mathbf{U}^{n+\theta}\right) \mathbf{V}^{n+1}=0
\end{aligned}
$$

In Eq. (44), $\gamma$ is a numerical parameter varying from 0 to 1. Eq. (45) is now multiplied by $\mathbf{D}$. Then Eq. (46) and the approximation $\mathbf{D} \mathbf{M}^{-1} \mathbf{G}=\mathbf{L}$, where $\mathbf{L}$ is the matrix of the Laplacian operator, lead to the following three steps:
$\frac{\mathbf{M}}{\delta t}\left(\widetilde{\mathbf{V}}^{n+1}-\mathbf{V}^{n}\right)+\mathbf{K}\left(\mathbf{U}^{n+\theta}\right) \widetilde{\mathbf{V}}^{n+\theta}+\gamma \mathbf{G}\left(\mathbf{U}^{n+\theta}\right) \mathbf{P}^{n}=\mathbf{F}^{n+\theta}$

$$
\delta t \mathbf{L}\left(\mathbf{U}^{n+\theta}\right)\left(\mathbf{P}^{n+\theta}-\gamma \mathbf{P}^{n}\right)=\mathbf{D}\left(\mathbf{U}^{n+\theta}\right) \widetilde{\mathbf{V}}^{n+1}
$$

$\frac{\mathbf{M}}{\delta t}\left(\mathbf{V}^{n+1}-\widetilde{\mathbf{V}}^{n+1}\right)+\mathbf{G}\left(\mathbf{U}^{n+\theta}\right)\left(\mathbf{P}^{n+\theta}-\gamma \mathbf{P}^{n}\right)=0$

The efficiency of this procedure is particularly appreciable in the resolution of the linear system. As a matter of fact, the classical convective term in an Eulerian formulation introduces the non-linearity of the system and the non-symmetry of matrix $\mathbf{K}$. With this fractional step procedure, $(\mathrm{dim}+1)$ symmetric matrices of size nare inverted instead of one matrix of size $n *(\operatorname{dim}+1)$, where 'dim' is the dimension of the problem, with a pre-conditioned conjugate gradient algorithm. With a complexity of the linear solver of $\mathrm{O}\left(n^{1.25}\right)$, an obvious gain of $\mathrm{CPU}$ time has been reached. In [36], this scheme is interpreted as the fully discrete counterpart of the projection method of the Chorin-Temam scheme at the semidiscrete level. Finally, the fractional step method possesses an intrinsic pressure stabilization property for

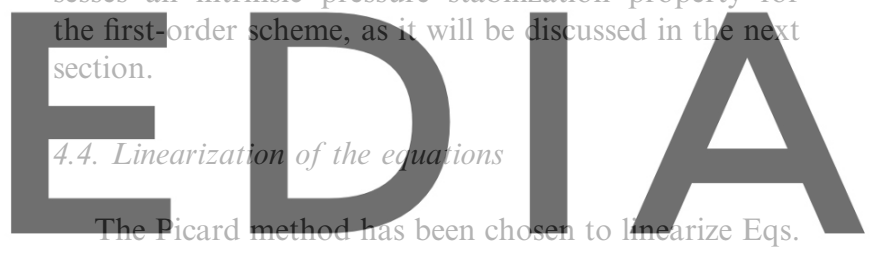

(47)-(49), as the Newton-Raphson implies too many ex-

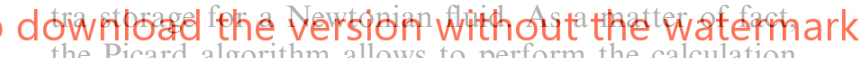

the Picard algorithm allows to perform the calculation in the deformed configuration taking into account the fully non-linear model with large rotations and finite strains. Linearizing with a Newton-Raphson method, as described in [37], produces many extra terms due to the fact that the constitutive equation is given in the deformed configuration as we will try to show.

For hyperelastic incompressible materials $[13,4]$ used in finite strains, problems are usually solved by the Newton or a Newton-like method, which involves the linearized form of balance equations. Considering an hyperelastic material, where the fundamental assumption is

$\mathbf{S}=\frac{\partial \Psi}{\partial \mathbf{E}}$

where $\mathbf{S}$ is the second Piola-Kirchhoff stress tensor, $\mathbf{E}$ the Green-Lagrange strain tensor and $\Psi$ the stored strain energy function, the linearization of the Principle of Virtual Work with respect to the displacement will give [1, p.338]

$\dot{f}^{\text {int }}=\int_{\Omega_{0}} \dot{\mathbf{P}}: \operatorname{grad}_{X}(\mathbf{N}) \mathrm{d} \Omega_{0}$ 
with $\mathbf{S}$ the first Piola-Kirchhoff stress tensor and $\mathbf{N}$ a test function. Using the relationship between the first and the second Piola-Kirchhoff stress tensor, the last equation reads

$\dot{f}^{\text {int }}=\int_{\Omega_{0}}\left(\dot{\mathbf{S F}}^{\mathbf{T}}+\mathbf{S} \dot{\mathbf{F}}^{\mathbf{T}}\right): \operatorname{grad}_{X}(\mathbf{N}) \mathrm{d} \Omega_{0}$

Introducing the elastic tensor $\mathbf{C}$

$\mathbf{C}=\frac{\partial \mathbf{S}}{\partial \mathbf{E}}=\frac{\partial^{2} \Psi}{\partial \mathbf{E}^{2}}$

Eq. (52) becomes

$\dot{f}^{\text {int }}=\int_{\Omega_{0}}\left(\mathbf{C} \dot{\mathbf{E}} \mathbf{F}^{\mathbf{T}}+\mathbf{S} \dot{\mathbf{F}}^{\mathbf{T}}\right): \operatorname{grad}_{X}(\mathbf{N}) \mathrm{d} \Omega_{0}$

It finally appears that the first term of the last equation, the material non-linearity, has been simplified thanks to the fundamental asumption of Eq. (50), for which the elastic tangent tensor puts in relation two quantities in the reference configuration. However, in the case of a fluid, the second Piola-Kirchhoff tensor is only known through its spatial counterpart $\sigma$, the Cauchy stress ten-
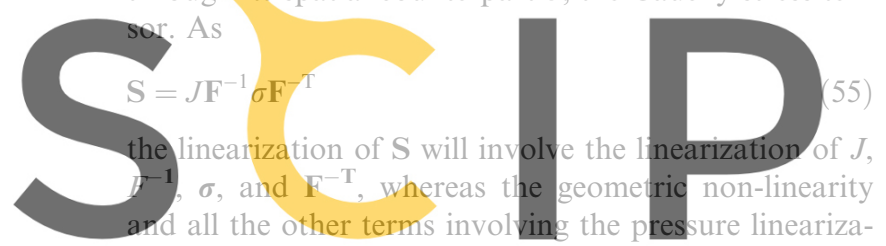

tion will present the same form. This difference is the

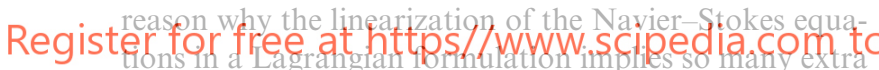
terms compared with the Picard linearization and an hyperelastic law formulation linearized with the Newton method.

Furthermore, during a time step, the mesh can suffer severe distortion due to the properties of a fluid to resist only to deformation rates. In order to preserve the positivity of the Jacobian, the time step is limited by the distance between a node and its normal projection on the opposite face weighted by the difference of velocity between these two points, so that the quick convergence of the Newton method for big time steps is unnecessary. A loop on the whole fractional step is performed. As the time step is limited to small values and the stabilization properties are limited to the first-order scheme in time, we considering the case $\gamma=0$. Writing as usual the index of the time step as the first superscript, and the one of the non-linear iteration as the second, the three steps of the algorithm read

$$
\begin{aligned}
& \left(\frac{\mathbf{M}}{\delta t}+\theta \mathbf{K}\left(\mathbf{U}^{n+\theta, i}\right)\right) \tilde{\mathbf{V}}^{n+1, i+1} \\
& =\mathbf{F}^{n+\theta}+\left(\frac{\mathbf{M}}{\delta t}-(1-\theta) \mathbf{K}\left(\mathbf{U}^{n}\right)\right) \mathbf{V}^{n}
\end{aligned}
$$

$$
\begin{aligned}
& \delta t \mathbf{L}\left(\mathbf{U}^{n+\theta, i}\right) \mathbf{P}^{n+\theta, i+1}=\mathbf{D}\left(\mathbf{U}^{n+\theta, i}\right) \widetilde{\mathbf{V}}^{n+\theta, i+1} \\
& \frac{\mathbf{M}}{\delta t}\left(\mathbf{V}^{n+1, i+1}-\widetilde{\mathbf{V}}^{n+1, i+1}\right)+\mathbf{G}\left(\mathbf{U}^{n+\theta, i}\right)\left(\mathbf{P}^{n+\theta, i+1}\right)=0
\end{aligned}
$$

\subsection{Coupled fluid-mechanics-thermal problems}

Now, the full coupled system temperature-displacement will be considered by introducing the Boussinesq approximation for incompressible fluids [43]

$\rho=\rho_{0}\left(1-\alpha\left(T-T_{0}\right)\right)$

where $\alpha$ is the volume/thermal expansion coefficient of the fluid, so that both previous schemes become with the fractional step
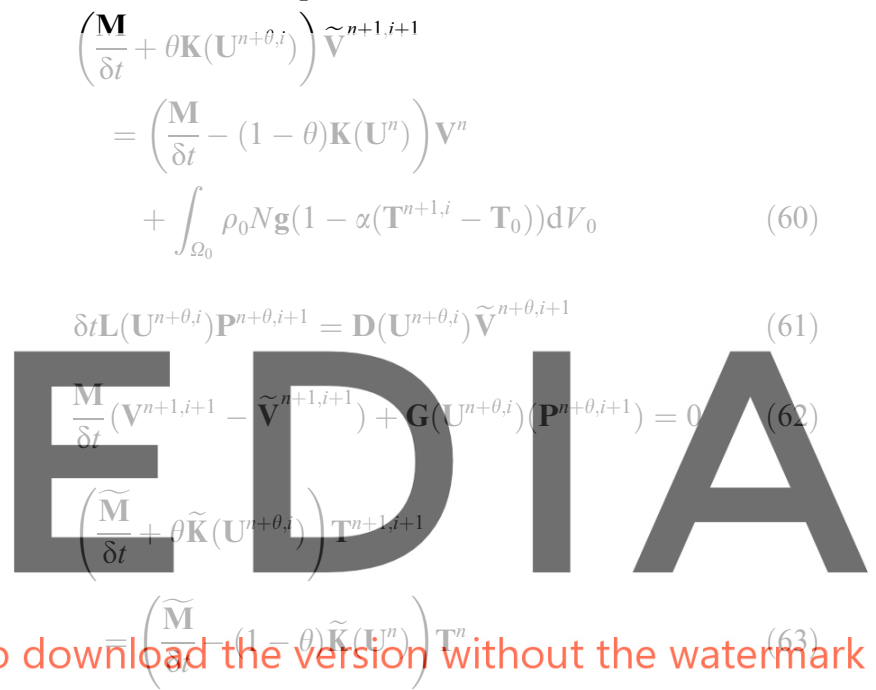

where $\widetilde{\mathbf{M}}$ and $\widetilde{\mathbf{K}}$ are deduced from $\mathbf{M}$ and $\mathbf{K}$ replacing the viscosity $\mu$ by the thermai conductivity $\kappa$, and the density $\rho$ by product of the density multiplied by the thermal capacity $C$, respectively. In order to smooth the strong gradients produced between two materials such as a mould and a melted metal in casting processes, a lumped mass matrix is used for the temperature. It reduces the under and overshoots produced numerically [7]. As boundary condition, a heat flux is taken into account on the free surface between the fluid and the external media. This is written in the classical form

$\Phi=h\left(T-T_{\text {ext }}\right)$

where $T_{\text {ext }}$ is the temperature of the external media and $h$ is the heat transfer coefficient between both considered media. This heat flux induces an extra term in matrix $\widetilde{\mathbf{K}}$ of Eq. (63).

\section{Computational features}

In this section, particular computational aspects of the method will be highlighted. In the first part, the 
pressure stability of the method is discussed. Then, the choice of reference configuration and its relationship with remeshing is presented. Finally, the particle features of the method are described.

\subsection{Pressure stabilization}

Because of the equal order interpolation, well-known problems appear in the computation of the pressure due to spurious pressure modes. This type of interpolation does not satisfy the discrete LBB condition

$\sup _{v_{h} \in V_{h}} \frac{b\left(v_{h}, q_{h}\right)}{\left\|v_{h}\right\| V_{h}} \geqslant \beta\left\|q_{h}\right\|_{Q_{h}} \quad \forall q_{h} \in Q_{h}, \beta>0$

where $V_{h}$ and $Q_{h}$ are, respectively, the finite element spaces of the velocity and the pressure, which leads to non-uniqueness of solution in pressure and possible checkerboard pressure modes $[3,20]$. However, as proved in [9], the first-order projection scheme used in the fractional step $(\gamma=0)$ brings in a stability term given by the difference between the 'discrete' and the continuous Laplacian operator, which depends on the time step. Eliminating $\widetilde{\mathbf{V}}$ from the fractional step as in [9], the con-
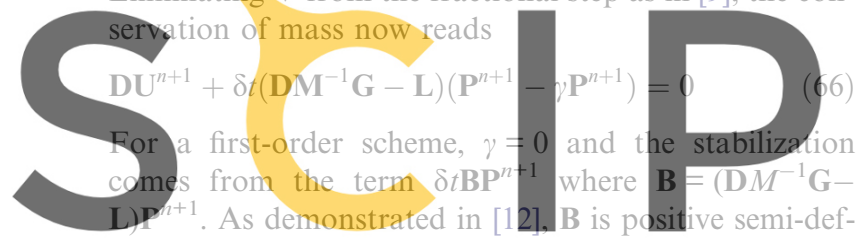

inite, provides a control on the orthogonals components

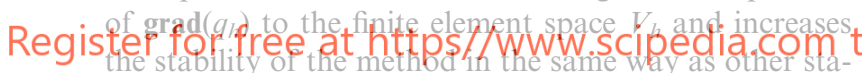

bilization methods [11]. As mentioned in [10], the inf-

sup condition is then weakened, as the space on which runs $V_{h}$ to fulfill (65) is then larger than $V_{h}$, which is satisfied using our equal-order interpolation.

As it has been shown, the stability term depends on the time step, so that for big time steps, the scheme is stable but dissipative, whereas for short time steps, the stability analysis does not provide any interesting bound. An appropriate choice for the time step is the explicit time step of the monolitical scheme. For an explicit scheme with convection dominant effects, which is our case, $\delta t_{\text {crit }}$ is basically equal to the Courant number $C=h / 2 U$ so that for a Courant number of one, the stability is guaranteed. As the nodes of the mesh cannot move more than one element length with the Lagrangian formulation, the values of $C \leqslant 1$ ensure that pressure stability can be easily achieved. However, it could happen that the condition on the Courant number could be verified locally in one part of the mesh and not in another because of the distortion of the mesh.

For second-order schemes in time, the stabilization effect of the fractional step is not efficient enough and other stabilization techniques must be used. The authors have developed a simple and general stabilized fractional step scheme, based on a Finite Calculus (FIC) formulation, ensuring pressure stabilization for both the first and second-order projection schemes $(\gamma=0$ and $\gamma=1)$ [30]. This formulation is based in the modification of the governing differential equations of the problem in evoking the balance of fluxes in a fluid domain of finite size. This introduces naturally additional terms that provide the necessary stabilization to the discrete equations obtained via the standard Galerkin finite element method. The final stabilized equations are

$r_{m_{i}}-\frac{h_{j}}{2} \frac{\partial r_{m_{i}}}{\partial x_{j}}=0$

for the momentum and

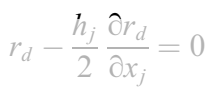

for the mass balance, where the residuals $r_{m_{i}}$ and $r_{d}$ are defined as

$r_{m_{i}}=\rho \frac{D u_{i}}{\mathrm{D} t}+\frac{\partial p}{\partial x_{i}}-\frac{\partial \sigma_{i j}}{\partial x_{j}}-\rho f_{i}$ (69)

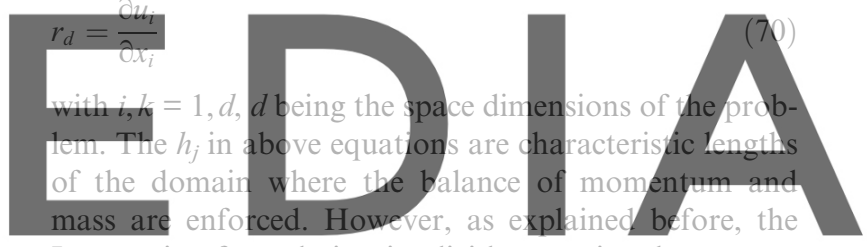

Lagrangian formulation implicitly contains the convec-

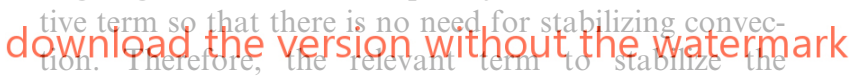
incompressibility constraint is given by Eq. (68). Details on how to obtain these equations can be found in [29] for an Eulerian formulation and in [31] for a Lagrangian formulation. The second-order scheme does not represent any difficulty with this stabilized scheme, but is not used in these examples as, as was already mentioned, the time step is short enough to provide an acceptable time accuracy.

\subsection{Reference configurations and (re)meshings}

The selection of the reference configuration $\Omega_{0}$ has not yet been discussed. Mainly, three choices are available for the reference configuration: the initial configuration (total Lagrangian formulation), the configuration at each time step, i.e. the deformed configuration (updated Lagrangian formulation), or the last known configuration, namely the one of the non-linear iteration. This choice is a priori completely independent of the type of non-linear problem considered.

However, as mentioned before, a fluid resists only to deformation rates, which implies to perform frequent remeshings in order to avoid the severe distortion of 
the elements. Really, only two possibilities are appealing, that is remeshing at each time step or at each nonlinear iteration. It could be observed that the choice of the reference configuration will decide the frequency of the meshing process. The results presented below have been performed with the first option, namely a mesh is generated once a convergent solution has been reached for each time step. Typically, between 2 and 4 iterations are necessary to obtain a strong convergence of order $10^{-3}$. Keeping the reference configuration constant within a time step allows to use the same shape functions and their derivatives during the non-linear iteration.

The numerical scheme of the last section can be applied for both formulations. If a new mesh is generated at each non-linear iteration, then it suffices to replace the gradient transformation by the identity matrix, and $\mathrm{J}$ by 1 and integrate on the current configuration. This method is presented in more detail in [24] and does not need the computation of the jacobian of the transformation as the current configuration is always the reference configuration.

The key point of the Lagrangian method lies on an accurate and powerful mesh generator. An efficient Del- aunay kernel is constructed with the reduced incremental method [18], verifying the star-shaped property of the cavity of each inserted point. Some acceleration procedures are required, as the use of a neighborhood grid, the transport of the centres of the circumdiscs and a special neighbor updating [17]. Obviously, a quasi linear relationship between time and data can only be obtained by the appropriate use of data structures, as described in [27] and [38]. The whole algorithm is summarized in Fig. 1.

\subsection{Particle methods}

In a previous work [23], the authors proposed a definition of a meshless method.

A meshless method is an algorithm that satisfies both of the following statements.

- the definition of the shape functions depends only on the node positions.

- the evaluation of the nodes connectivity is bounded in time and it depends exclusively on the total number of nodes in the domain.
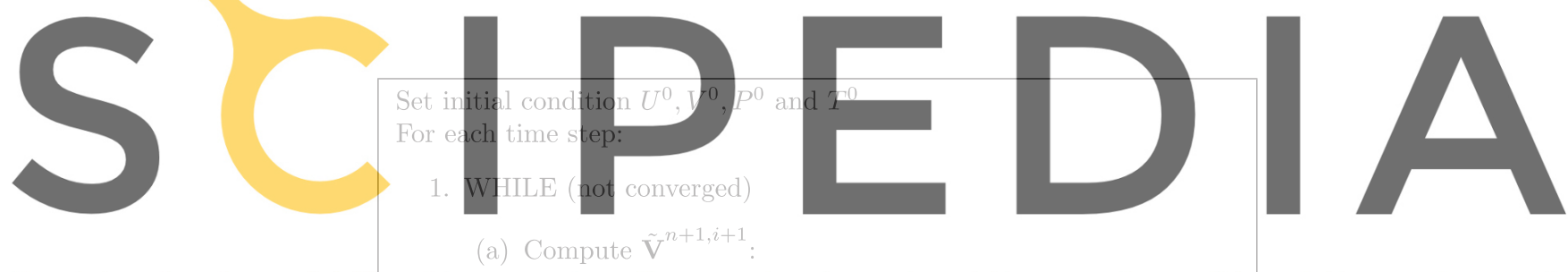

Register for free at https//www.schpedia.com to download the version without the watermark

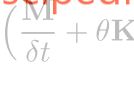

$$
+\int_{\Omega_{0}} \rho_{0} N \mathbf{g}\left(1-\alpha\left(\mathbf{T}^{n+1, i}-\mathbf{T}_{0}\right)\right) d V_{0}
$$

(b) Compute $\mathbf{P}^{n+\theta, i+1}$ :

$$
\delta t \mathbf{L}\left(\mathbf{U}^{n+\theta, i}\right) \mathbf{P}^{n+\theta, i+1}=\mathbf{D}\left(\mathbf{U}^{n+\theta, i}\right) \tilde{\mathbf{V}}^{n+\theta, i+1}
$$

(c) Compute $\mathbf{V}^{n+1, i+1}$ :

$$
\frac{\mathbf{M}}{\delta t}\left(\mathbf{V}^{n+1, i+1}-\tilde{\mathbf{V}}^{n+1, i+1}\right)+\mathbf{G}\left(\mathbf{U}^{n+\theta, i}\right)\left(\mathbf{P}^{n+\theta, i+1}\right)=0
$$

(d) Compute $\mathbf{T}^{n+1, i+1}$ :

$$
\left(\frac{\tilde{\mathbf{M}}}{\delta t}+\theta \tilde{\mathbf{K}}\left(\mathbf{U}^{n+\theta, i}\right)\right) \mathbf{T}^{n+1, i+1}=\left(\frac{\tilde{\mathbf{M}}}{\delta t}-(1-\theta) \tilde{\mathbf{K}}\left(\mathbf{U}^{n}\right)\right) \mathbf{T}^{n}
$$

2. If converged, update the mesh nodes with the Newmark scheme

3. Generate a new mesh

4. Compute the new time step

Fig. 1. Thermally coupled incompressible flow solved with the Lagrangian algorithm. 
In this work, the first statement is not verified as usual finite element shape functions are used, which depend on the element geometry and not on the nodes position. However, the second statement, which constitutes the most important fact of the meshless methods is verified through an efficient Delaunay triangulation. In the PFEM, the most salient characteristic is that all the information is transferred through the particles. At each time step, each particle carries its own information like its type of material and the value of all the unknowns of the considered problem, here the velocity, pressure and temperature. Furthermore, as the computational domain is also unknown, the decision on the choice of the geometrical domain in the analysis will exclusively rely on the boundary definition and the node information. This last feature is typical of the meshless methods, where the physical properties are directly associated with the type of material of the node.

The boundary definition follows the work of [24]. In particle methods, the connectivity between each node is

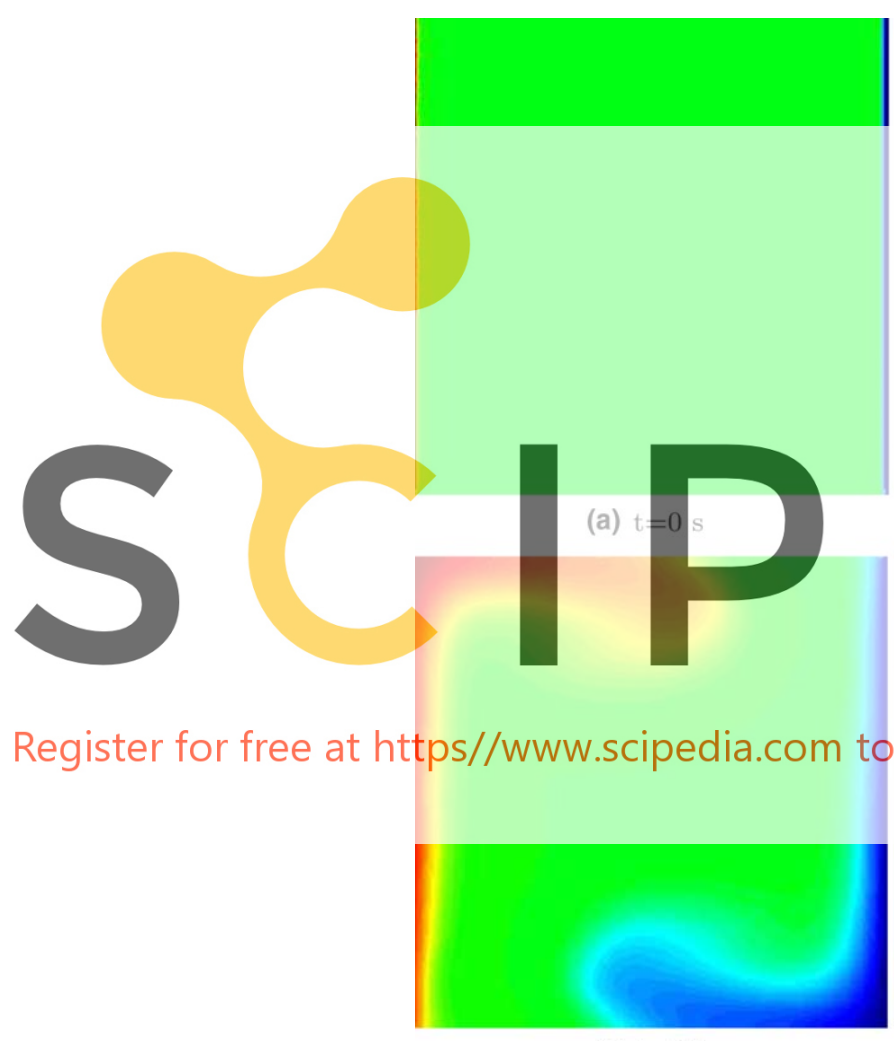

(c) $\mathrm{t}=58 \mathrm{~s}$

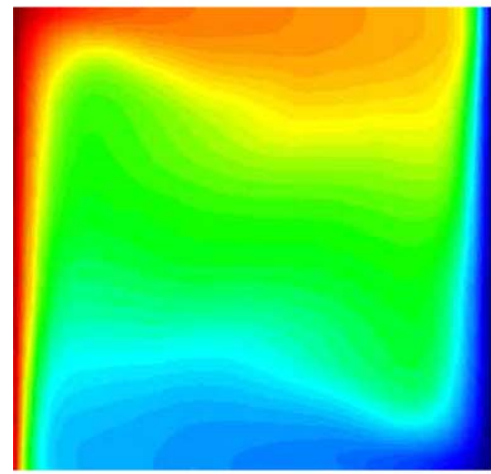

(e) $\mathrm{t}=138 \mathrm{~s}$

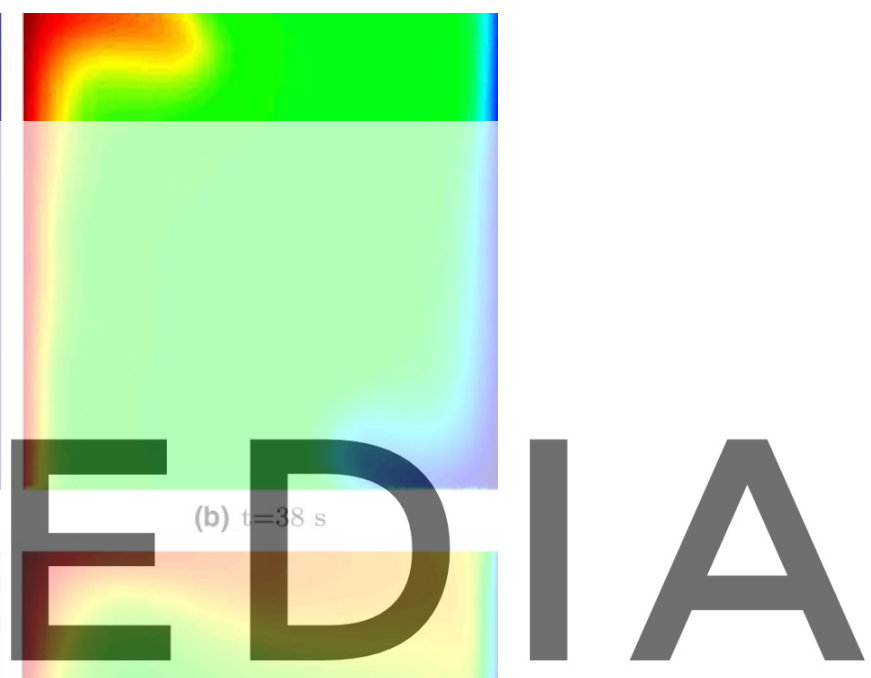

download the version without the watermark

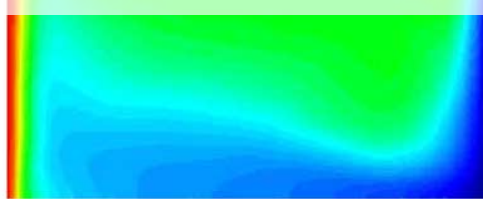

(d) $\mathrm{t}=98 \mathrm{~s}$

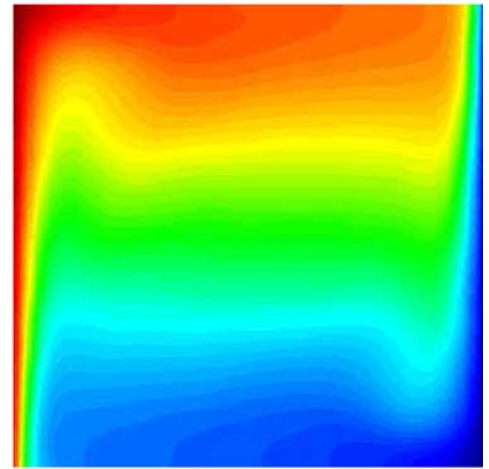

(f) $\mathrm{t}=398 \mathrm{~s}$

Fig. 2. Temperature distribution in a closed cavity for $\theta \in\{19.5 ; 20.5\}$. 
constructed at each time step. This connection gives plenty of freedom to define the system to be studied, the inner connections, and the possible multiple boundaries of the system. As the system is constantly moving, the location of the boundaries must also be redefined accordingly. In order to characterize the boundaries, the alpha shape method [16] is used, which could be formulated as

Given a particle distribution depending on $h(x)$, where $h(x)$ is the minimum distance between two particles, all particles on an empty sphere with a radius $r(x)$ larger than $\alpha h(x)$ are considered as boundary particles. In this criterion, $\alpha$ is a parameter close to, but greater than one.

Respect to the inner connections, an interesting feature of this method happens when a particle is suddenly separated from the rest of the body. In this case, the particle is considered as dimensionless and thermally isolated from the rest of the particles. Hence two options are possible: whether it will conserve the same temperature than the last time step, or the temperature of the

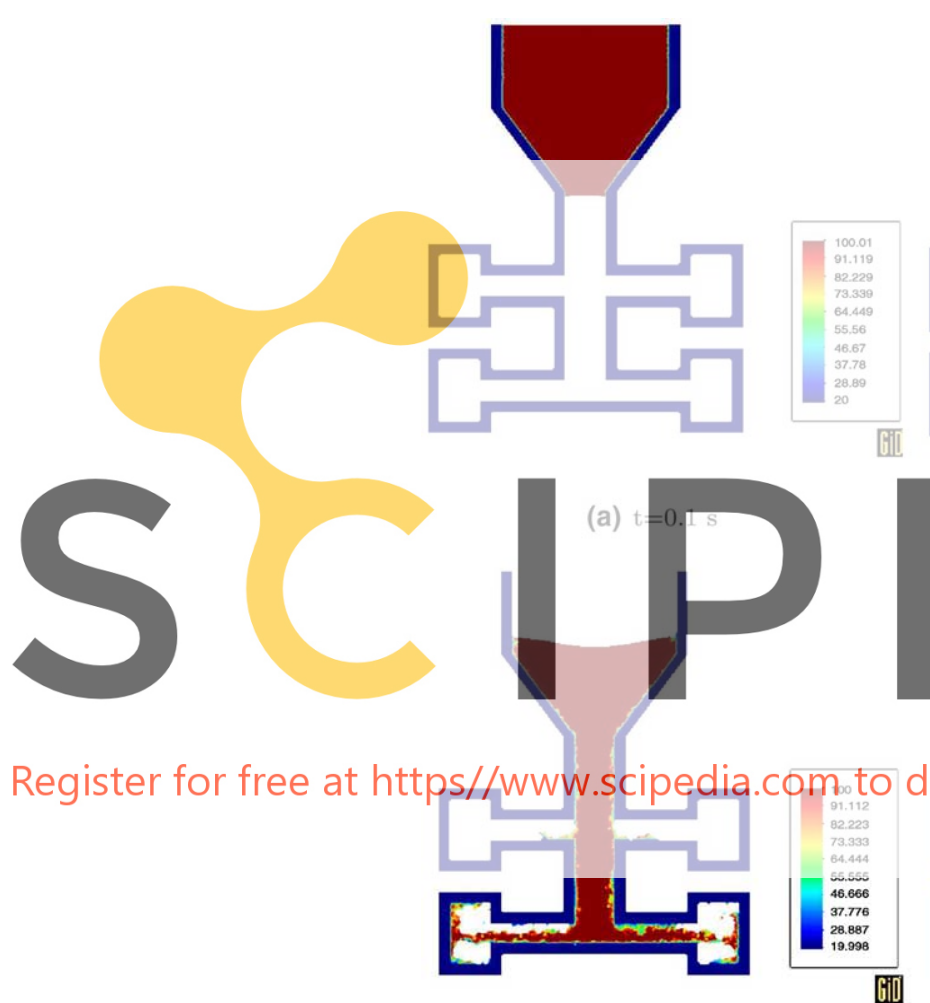

(c) $\mathrm{t}=1.5 \mathrm{~s}$

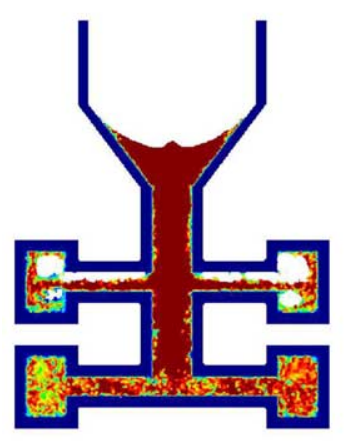

(e) $\mathrm{t}=2.3 \mathrm{~s}$
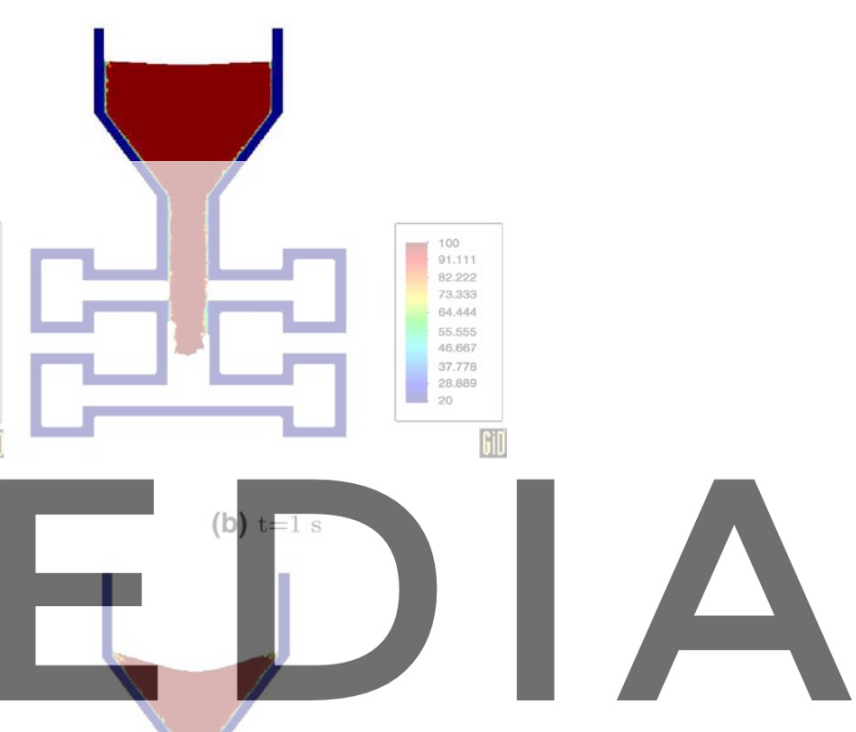

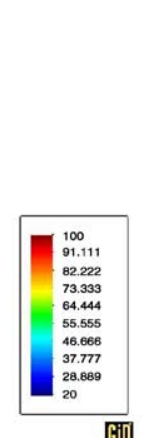

[1010

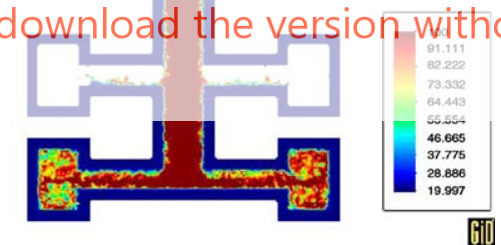

(d) $\mathrm{t}=1.8 \mathrm{~s}$

阿

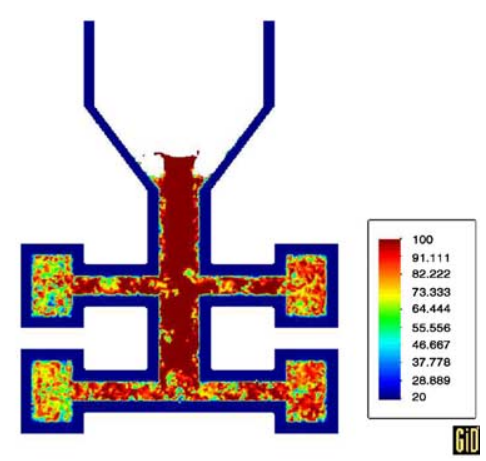

(f) $\mathrm{t}=2.9 \mathrm{~s}$

Fig. 3. Temperature distribution at different instants during the mould filling. 
external media will be imposed to this particle. In both cases, this particle will follow its motion under gravity forces at a constant temperature.

Finally, as the particles are constantly convected, they could get closer and closer, until they superimpose. During this process, a particle has been lost which induces a loss of information of the studied variables. Moreover, the mesh quality diminishes as quick as the time step. To avoid this problem, a background grid (bucket sort) is used to filter the relative position of the points between each other. Thus, if a particle is closer than a certain tolerance, it is taken away and reintroduced in another part with low density of particles, in order to homogenize the information. A standard linear projection of the problem variables is used to transfer the information from the current grid to the new particle. This type of problem appears frequently in closed domains were the fluid particles do not have possibilities to cover a large space, as it will be noted in the numerical results presented next.

\section{Numerical examples}

In this section, four 2-D numerical experiments are presented to illustrate the viability of the PFEM. The first example has been chosen to validate the method by comparison with previous established numerical results obtained for the same problem using an Eulerian formulation. The second example describes a typical free surface problem with contact on a solid. The third and fourth examples involve a complete thermo-mechanical coupling with the classical Rayleigh-Bénard instability. However, the last example introduces a not so classical free-rigid boundary condition.

\subsection{Thermal convection with the Boussinesq approximation in a cavity}

This example is a classical benchmark for Eulerian formulations. The fluid is inside a cavity, the left wall is heated isothermally to $20.5^{\circ} \mathrm{C}$, and the right wall is heated isothermally to $19.5^{\circ} \mathrm{C}$, the other sides being adiabatic. The fluid is initially at $20{ }^{\circ} \mathrm{C}$, which is the reference temperature. The Rayleigh number, defined as

$R a=\frac{\alpha g \Delta T L^{3}}{v \kappa}$

has been chosen as in [39], and is equal to $10^{6}$. The Prandtl number, defined as

$\operatorname{Pr}=\frac{v}{\kappa}$

is equal to 1 . The cavity is a square, as in [39]. The mesh is composed of 16,400 nodes during the whole analysis,
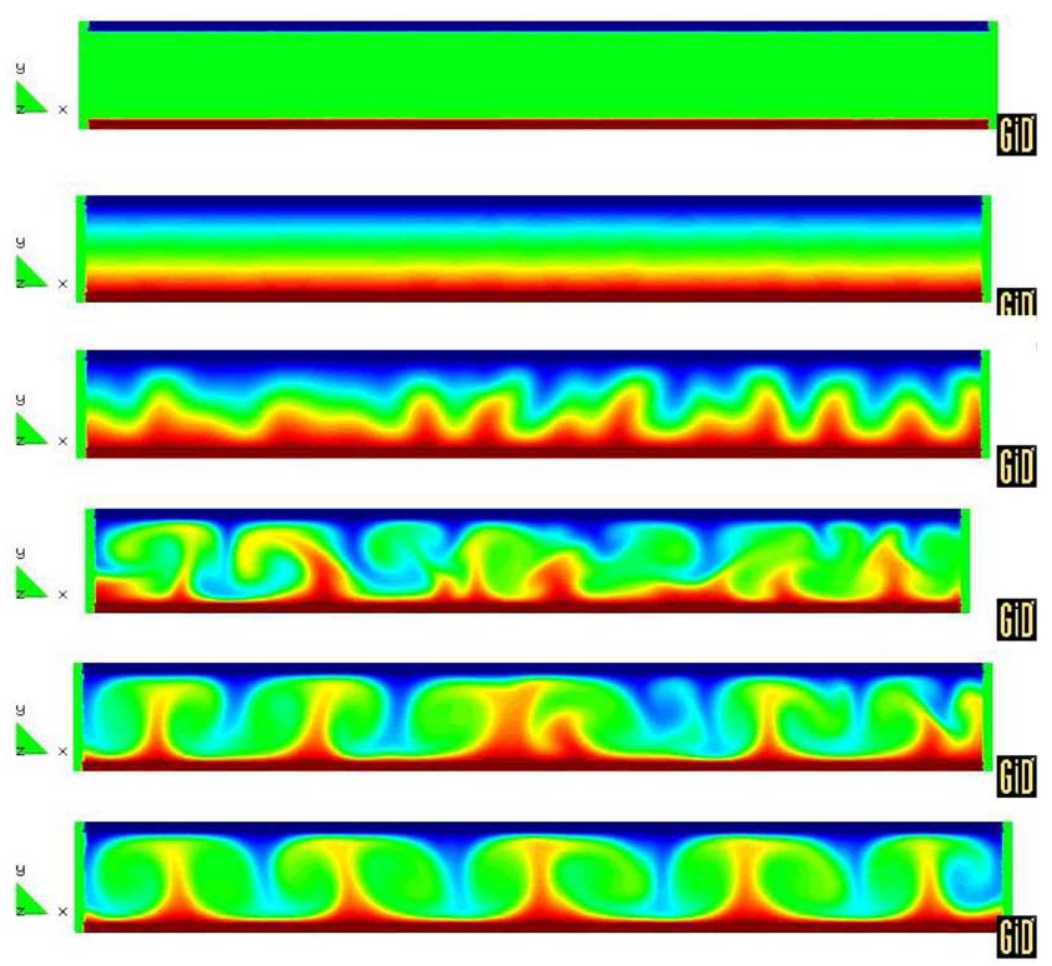

Fig. 4. Temperature distribution for the rigid-rigid Rayleigh-Bénard instability for $t=\{0.1,38,78,108,158,400\}$ and $\theta \in\{19 ; 21\}$. 
and 32,000 elements at the beginning, 2/3 for the fluid part. As explained in the last section, if a node is too close to another one, it is removed and placed in another part, but the total number of nodes remains constant.

The temperature and velocity distributions are presented in Fig. 2 at different time values. The results are in perfect agreement with the one obtained by Strada and Heinrich [39]. It should be noticed that these kind of examples are typical Eulerian examples, the configuration being particularly bad for the Lagrangian approach computationally. As a matter of fact, as the cavity is closed, there is much more contact with the wall than in usual free surface problems, and the space to fill for the element is much more reduced. However, the results obtained here demonstrate the validity of the method, even in this difficult case.

\subsection{Mould filling}

This example represents a mould filling by water at $100^{\circ} \mathrm{C}$. The mould initially has a temperature of $20^{\circ} \mathrm{C}$. The coupling between the mechanical and thermal parts is only partial due to the mesh movement, but the velocity is not influenced by the temperature. An external flux is applied with an external temperature of $10{ }^{\circ} \mathrm{C}$ on all the boundaries, namely the mould boundary as well as the fluid boundary. The mesh is initially formed by 12,000 nodes and 23,000 elements. We represent a mould with two floors, which is often the case in the industry, to emphasize the problem for the fluid to follow the main channel and not to enter directly in the first floor, which is a classical difficulty for Eulerian methods with the use of pseudo-concentration. In the Lagrangian formulation, the motion of the fluid is naturally driven by the gravity forces so that this kind
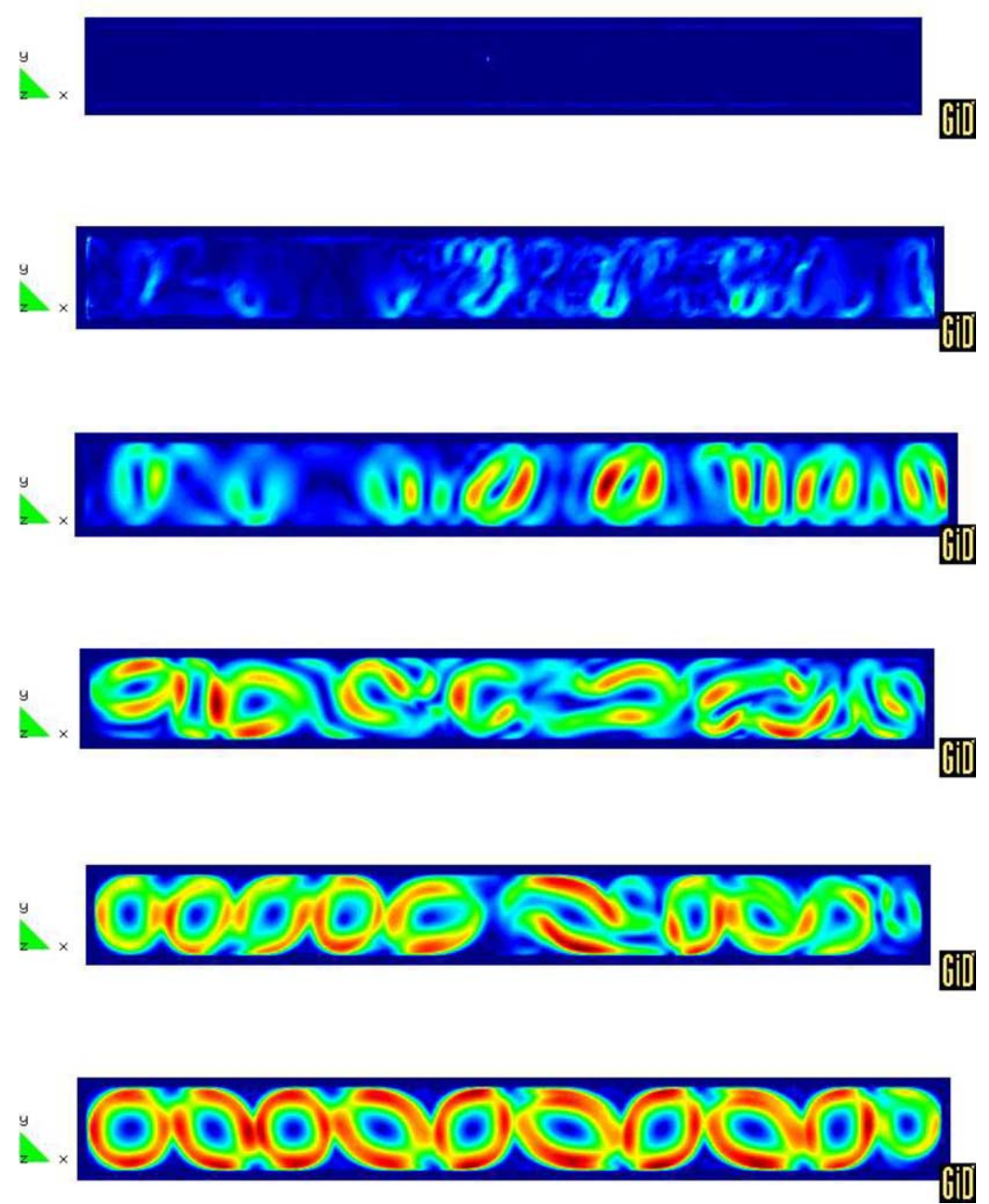

Fig. 5. Velocity norm for the rigid-rigid Rayleigh-Bénard instability for $t=\{0.1,38,78,108,158,400\}$. 
of examples do not really represent a difficulty. The contact is explicitly treated by the remeshing. The only potential problem could be to choose a time step too big so that the particle goes through the wall without having created an element of contact with it. However, the distortion of the mesh can not imply such non-acceptable time steps.

Numerical results are shown in Fig. 3. It can be appreciated how the fluid breaks against the sides of the mould and produces in the four squares a mixing by convection which diffuses quickly the temperature. The wave created on the upper part of the fluid when the mould is completely filled, is depicted in the last picture. Despite of the remeshing, the boundary definition, and the reintroduction of nodes, the global filling possesses a clear symmetry. There is no loss of mass during the process and the second floor really begins to fill when the first floor is already filled.

\subsection{The Rayleigh-Bénard instability}

This example is a classical problem of hydrodynamic instabilities, see [14] and [21] for a good description of it. The problem considered is a fluid initially at rest, which is heated from below. Assuming an infinitesimal perturbation of temperature, the Boussinesq approximation will induce a vertical movement towards the cooler region for the hotter lower part and viceversa, which will reinforce the initial perturbation. Given a critical Rayleigh number, the state of the flow will depend on the fact that its Rayleigh number is lesser or greater than the critical value. For $R a<R a_{c}$, no convection will occur and the flow is subcritical. If $R a>R a_{c}$, the instability will begin and produce the convection. The flow is then supercritical. For a slightly supercritical Rayleigh number, after passing through successive bifurcations, a steady state is reached as illustrated in [42], p 82. By
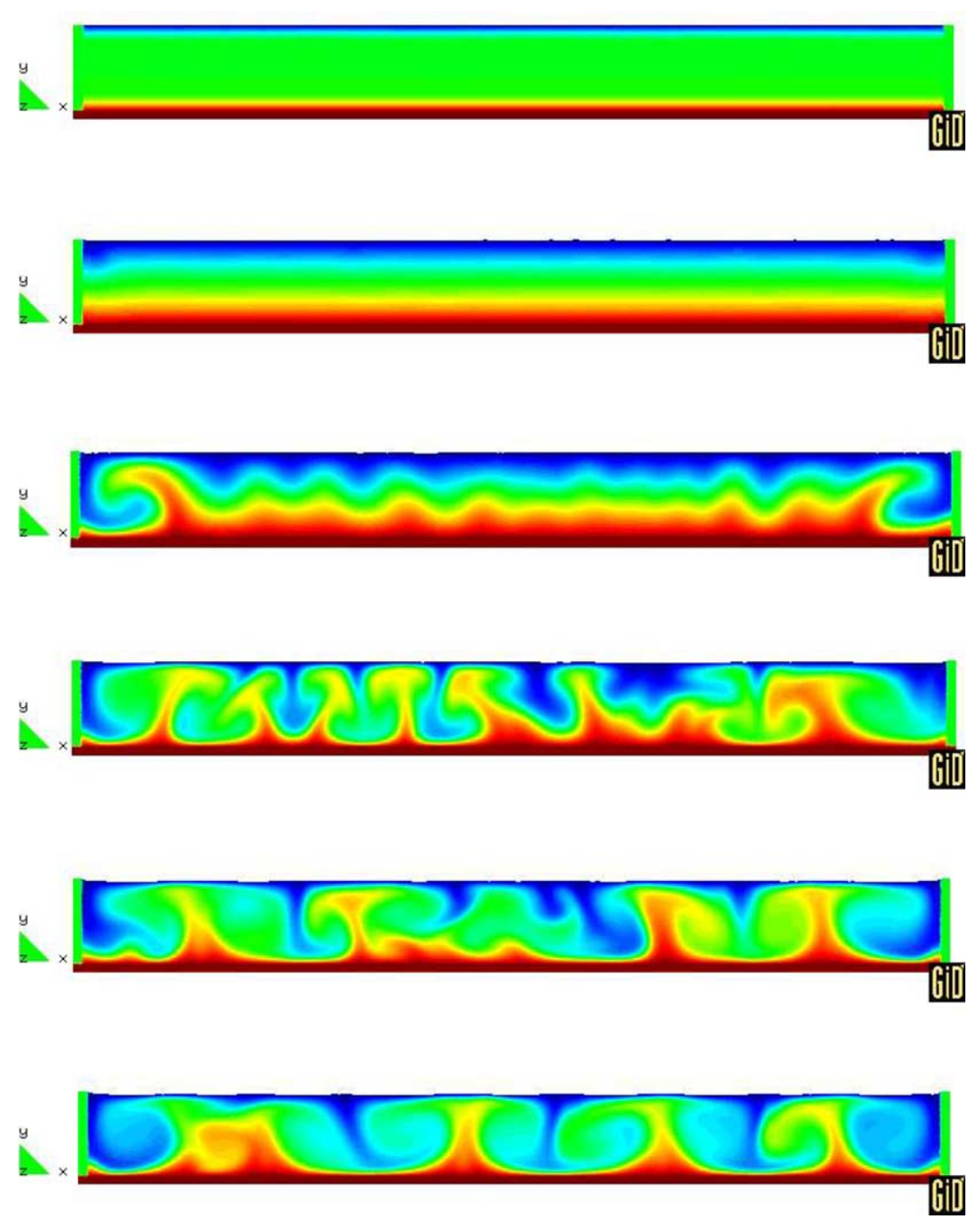

Fig. 6. Temperature distribution for the free-rigid Rayleigh-Bénard instability for $t=\{4,38,78,108,158,400\}$ and $\theta \in\{19 ; 21\}$. 
increasing the Rayleigh number, other bifurcations occur after the primary bifurcations, according to the Prandtl number, as depicted experimentally in [14], until reaching a turbulent flow.

By linearizing the mass conservation equation and the Navier-Stokes equations coupled with the heat equation in an Eulerian formulation, and by introducing a decomposition in normal modes, as performed in [15], a stability study leads to a solution of the form

$w=W(z) f(w, y) \mathrm{e}^{s t}, \quad T=T(z) f(x, y) \mathrm{e}^{s t}$

where $s$ is a complex number representing the eigenvalue of the mode, $w$ is the vertical component of the velocity, and $f$ an unknown function. The solution of the linearized equation with non-physical free-free boundaries has the form

$W_{n}=A \sin (n \pi z) \quad(n=1,2 \ldots)$
The sign of the real part of s will decide of the stability of the flow. By solving the last equations with $s=0$, and minimizing the Rayleigh number with respect to the wave number, it is possible to find the critical Rayleigh number, analytically for the free-free boundary condition, and numerically for the rigid-rigid and free-rigid boundary condition, as explained in [15] and [6]. For the rigid-rigid case, $R a_{c}=1708$.

In this numerical example, the bottom is heated isothermally at $21^{\circ} \mathrm{C}$, the top at $19{ }^{\circ} \mathrm{C}$, and the reference and initial temperature of the fluid is $20^{\circ} \mathrm{C}$. The sides are adiabatic and $R a=10^{5}$ and $\operatorname{Pr}=10^{-1}$. The flow is then supercritical. The numerical results of Fig. 5, which represent the norm of the velocity clearly depict the typical cell pattern observed experimentally when one horizontal side is much shorter than the other. These cells form rolls rotating in opposite direction
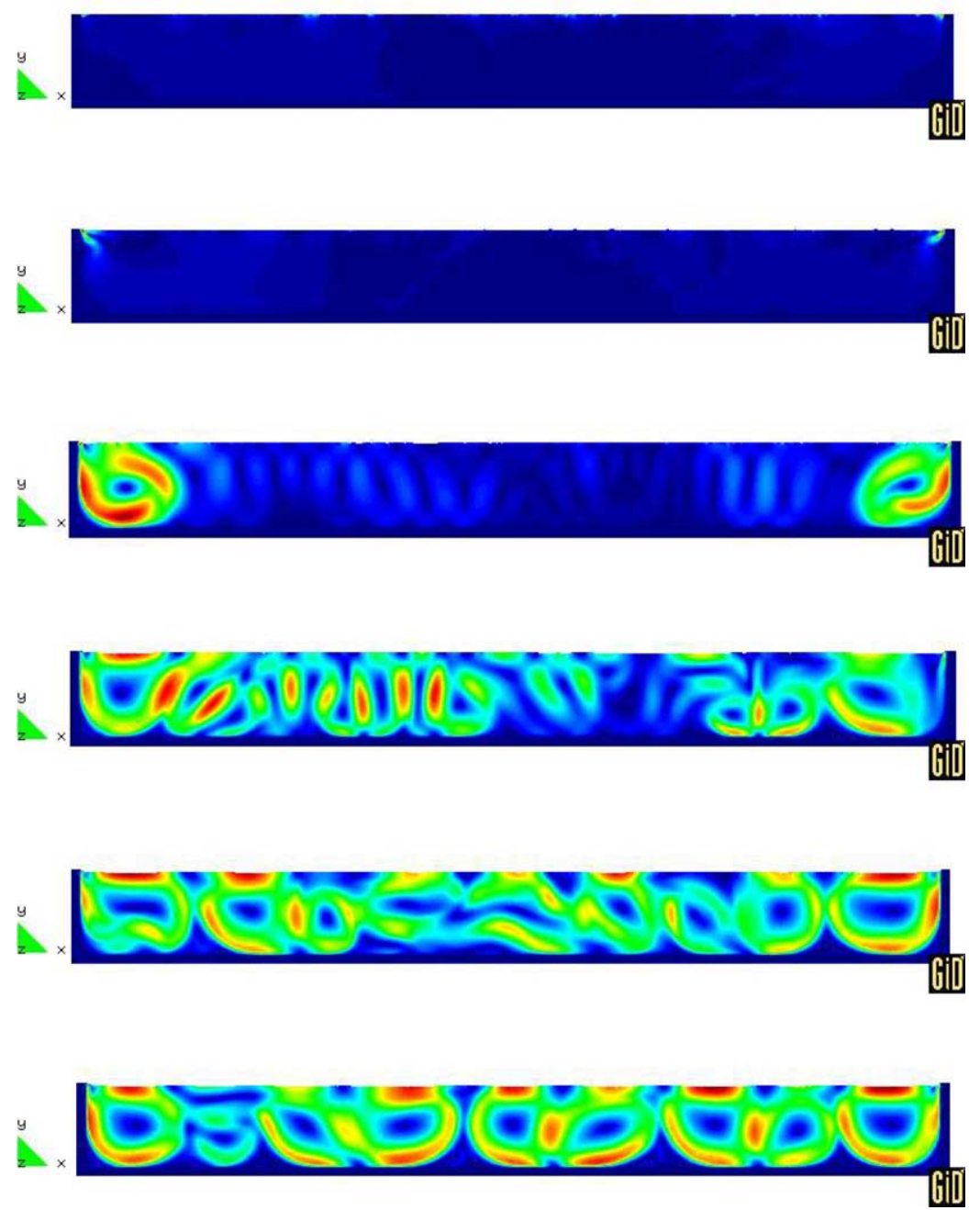

Fig. 7. Velocity norm for the free-rigid Rayleigh-Bénard instability for $t=\{4,38,78,108,158,400\}$. 
for neighboring rolls, along the shortest side. However, what can not be well appreciated on the picture and is particularly remarkable, is that a quasi-steady state is reached, with a periodic oscillation of the temperature and the cells. This phenomenon is described in detail in [26] by considering moderately non-linear convection, and it is to be noticed that the numerical results coincide with experimental ones for low Prandtl number, which is exactly the situation here. The rolls oscillate as depicted in [8]. It is interesting to notice the interaction between the different cells before the quasi-steady state. The supercritical state could be observed through the approximate regularity of the cells at the last time step. The results are in agreement with [5] in the sense that the solution is time dependant for such a high Rayleigh number value. Fig. 4 represents the temperature distribution at different moments and corresponds to the theoretical expected result of a supercritical flow.

\subsection{The Rayleigh-Bénard instability with free surface}

This example is rarely treated as it combines the difficulty of the thermal convection with the detection of free surfaces. The problem considered here does not take into account any surface tension, a phenomenon associated to the hydrodynamical instability of BénardMarangoni. Here, the coupling between temperature and displacement produces the instability, and circular cells are expected, compared to the appearance of hexagonal cells in the case of the Bénard-Marangoni instability [43].

The same conditions as in the previous example are chosen. A temperature of $19{ }^{\circ} \mathrm{C}$ is imposed on the free surface of the fluid. The same instants as above are reported to compare with the rigid-rigid boundary case. To follow the discussion on the critical Rayleigh number, the boundary conditions considered here imply $R a_{c}=1101$ theoretically so that the numerical experiment has a Rayleigh number far beyond $R a_{c}$. As seen in Fig. 7, the quasi-steady state has not been reached, due to the high Rayleigh number value. Some parts of the domain present a quasi static behaviour, but some cells are unstable. The rolls oscillate as in the preceeding example but some of the cells appear and disappear which is well observed at the last time step for the cells in the middle of the left part of the experiment. If the calculation of the critical Rayleigh number for the free-rigid case is well-known, it has not been possible to find in the literature theoretical results about the pattern of the cells for this kind of boundary conditions. Fig. 6 represents the temperature distribution which contains the same unstable behaviour as the velocity. Fig. 8 represents a detail of two cells with their temperature, norm of the velocity and velocity field.

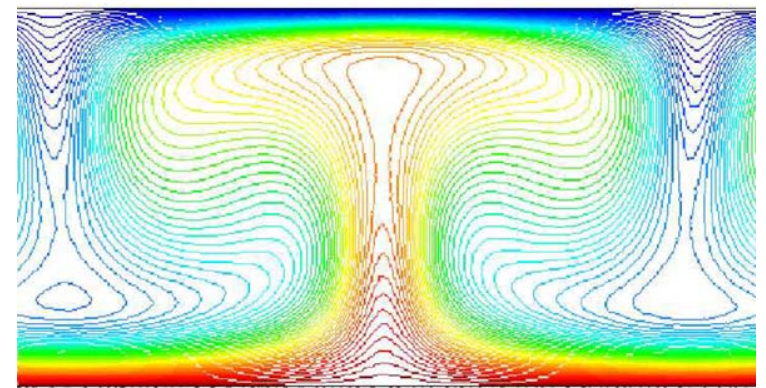

(a) Temperature isolines

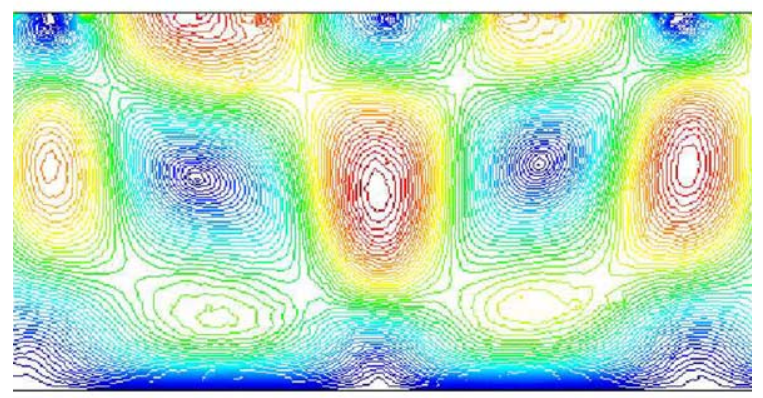

(b) Velocity norm isolines

Fig. 8. Detail of two cells for the free-rigid Rayleigh-Bénard instability.

\section{Conclusion}

A fully non-linear Lagrangian formulation to solve incompressible fluids with thermal convection and free surfaces has been presented in this paper. Details of the mathematical model and the fractional step algorithm chosen for solving the discrete equations in time have been highlighted. The Lagrangian formulation allows to avoid the instability problem induced by the convection terms, typical of the Eulerian formulation. This is done by transferring the convection to the motion of the nodes, and its inherent difficulty to the mesh generation. However, with the last improvements realized in the mesh generation field, it is now feasible to incorporate the mesh generation step as an intrinsic and fundamental part of the calculation process.

In the numerical examples presented, the method has proved to be efficient even in an Eulerian context, where the confined spaces produce difficulties at the mesh generation level. From a computational standpoint, only symmetric matrices are inverted, so that the associated linear equations can be solved in a very efficient manner with a classical pre-conditioned conjugate gradient algorithm. Furthermore, the fractional step approach has proved to be an efficient procedure for solving accurately the Lagrangian flow equations.

Future works will cover the extension of thermal results to three dimensional problems, and especially the 
introduction of solidification during mould filling in casting problems. An other interesting feature of the PFEM to be investigated is its ability to introduce particles at given location with a given density in order to better capture special features of the solution.

\section{Acknowledgement}

Thanks are given to Dr. Facundo Del Pin for all its support and experience in the Lagrangian methods applied to fluids during the development of the present method. The visualization of the numerical results was performed with the GID pre/postprocessing system developed at CIMNE (www.gidhome.com).

\section{References}

[1] Belytschko T, Liu WK, Moran B. Nonlinear finite elements for continua, and structures. Wiley; 2000.

[2] Belytschko T, Liu Y, Gu L. Element free Galerkin methods. Int J Numer Methods Eng 1994;37:22956.

[3] Brezzi F. On the existence and uniqueness of saddle-point problem arising from Lagrangian multipliers. R.A.I.R.O. 1974;8:129-51.

[4] Brink U, Stein R. On some mixed finite element methods for incompressible and nearly incompressible finite elasticity. Computat Mech 1996;19:105-19.

[5] Busse FH. Non linear properties of thermal convection. Rep. Prog. Phys. 1978;41:1929-67.

[6] Chandrasekar S. Hydrodynamic and hydromagnetic stability. Oxford: Clarendon-Press; 1961.

[7] Chiumenti M. Constitutive modeling and numerical analysis of thermo-mechanical phase change systems. PhD thesis, UPC; 1998.

[8] Clever RM, Busse FH. Transition to time dependant convection. J Fluid Mech 1974;65:625-45.

[9] Codina R. Pressure stability in fractional step finite element methods for incompressible flows. J Computat Phys 2000;190:1579-99.

[10] Codina R, Blasco J. E finite element formulation for the stokes problem allowing equal velocity-pressure interpolation. Comput Methods Appl Mech Eng 1997;143:373-91.

[11] Codina R, Vazquez M, Zienkiewicz OZ. A general algorithm for compressible and incompressible flow, Part I: The split, characteristic-based scheme. Int J Numer Methods Fluids 1995;20:869-85.

[12] Codina R, Vazquez M, Zienkiewicz OZ. A general algorithm for compressible and incompressible flow, Part III: the semi-implicit form. Int J Numer Methods Fluids 1998;27:13-32.

[13] De S, Bathe KJ. The method of finite sphere with improved numerical integration. Comput Struct 2001;79:2183-96.

[14] Drazin PG. Introduction to Hydrodynamic Stability. Cambridge University Press; 2002.

[15] Drazin PG, Reid WH. Hydrodynamic Stability. Cambridge University Press; 1981.
[16] Edelsbrunner H, Mücke EP. Three-dimensional alpha shapes. ACM Trans Graph 1994;13(1):43-72.

[17] George PL, Borouchaki H. Aspects of 2-d Delaunay mesh generation. Int J Numer Methods Eng 1997;40: 1957-75.

[18] George PL, Borouchaki H. Triangulation de Delaunay et maillage. Hermes; 1998.

[19] Gingold RA, Monaghan JJ. Smoothed particle hydrodynamics, theory and application to non-spherical stars. Mon Nat R Astr Soc 1977;181:375-89.

[20] Girault V, Raviart PA. Finite element methods for NavierStokes equation. Berlin: Springer; 1986.

[21] Guyon E, Hulin JP, Petit L. Physical hydrodynamics. Oxford University Press; 2001.

[22] Hughes TJR. Analysis of transient algorithms with particular reference to stability behaviour. In: Belytschko T, Hughes TJR, editors. Computational methods for transient analysis. Amsterdam: North-Holland; 1983. p. 67-155.

[23] Idelsohn SR, Oñate E, Calvo N, Del Pin F. The meshless finite element method. Int $\mathbf{J}$ Numer Methods Eng 2003;58(6):893-912.

[24] Idelsohn SR, Oñate E, Del Pin F. A Lagrangian meshless finite element method applied to fluid-structure interaction problems. Comput Struct 2003;81:655-71.

[25] Idelsohn SR, Oñate E, Del Pin F. The particle finite element method: a powerful tool to solve incompressible flows with free-surfaces and breaking waves. Int $\mathbf{J}$ Numer Methods Eng 2004;61(7):964-89.

[26] Koschmieder EL. Bénard cells and Taylor vortices. Cambridge University Press; 1993.

[27] Löhner R. Some useful data structures for the generation of unstructured grids. Commun Appl Numer Methods 1988:123-35.

[28] Marsden JE, Hughes TJR. Mathematical foundations of elasticity. New York: Dover; 1983.

[29] Oñate E. A stabilized finite element method for incompressible viscous flow using a finite increment calculus formulation. Comput Methods Appl Mech Eng 2000;182:355-70.

[30] Oñate E, Garcia J, Bugeda G, Idelsohn SR. A general stabilized formulation for incompressible fluid flow using finite calculus and the finite element method. In: Periaux J, Chamption D, Pironneau O, Thomas Ph, editors. Towards a new fluid dynamics with its challenges in aeronautics. Barcelona, Spain: CIMNE; 2002.

[31] Oñate E, Idelsohn SR, Del Pin F. Fully Lagrangian formulation for fluid-structure interaction problems using the finite element method and finite calculus. In: Mathisen KM, Kvamsdal T, Okstad KM, editors. Computational mechanics theory and practice. Barcelona, Spain: CIMNE; 2003.

[32] Oñate E, Idelsohn SR, Del Pin F, Aubry R. The particle finite element method: an overview. Int $\mathbf{J}$ Computat Methods 2004;1(2):267-307.

[33] Oñate E, Idelsohn SR, Zienkiewicz OC, Taylor RL. A finite point method in computational mechanics, application to convective transport and fluid flow. Int $\mathbf{J}$ Numer Methods Eng 1996;39:3839-86.

[34] Oñate E, Idelsohn SR, Zienkiewicz OC, Taylor RL, Sacco C. A stabilized finite point method for analysis of fluid 
mechanics problems. Comput Methods Appl Mech Eng 1996;39:315-46.

[35] Ogden RW. Non-linear elasticity. New York: Dover; 1997.

[36] Quarteroni A, Saleri F, Veneziani A. Factorization methods for the numerical approximation of Navier-Stokes equations. Comput Methods Appl Mech Eng 2000;188:505-26.

[37] Radovitzky R, Ortiz M. Lagrangian finite element analysis of Newtonian fluid flows. Int $\mathbf{J}$ Numer Methods Eng 1997;43:607-19.

[38] Samet H. The design and analysis of spatial data structures. Reading, MA: Addison-Wesley; 1990.
[39] Strada M, Heinrich JC. Heat transfer rates in natural convection at high Rayleigh numbers in rectangular enclosures: a numerical study. Numer Heat Transfer 1982;5:81-93.

[40] Temam R. Navier-Stokes equations. Amsterdam: NorthHolland; 1984.

[41] Temam R. Navier-Stokes equations and nonlinear functional analysis. Philadelphia: SIAM; 1995.

[42] Van Dyke M. An album of fluid motion. Stanford, CA: The Parabolic Press; 1989.

[43] R.Kh. Zeytounian, Il y a cent ans...et aujourd'hui l'approximation de boussinesq. Matapli, 71, April 2003. 